\title{
In Situ Biasing of Conductive Bridge Resistive Memory Devices Observed in a Transmission Electron Microscope
}

\author{
Remy Berthier ${ }^{1,2}$, Cécile Nail ${ }^{1,2}$, Catherine Carabasse ${ }^{1,2}$, Gabriel Molas $^{1,2}$ and David Cooper ${ }^{1,2}$ \\ 1. Univ. Grenoble Alpes, Grenoble, France \\ 2. CEA-LETI, MINATEC Campus, Grenoble, France
}

Resistive memories are considered as the most promising candidate for the replacement of conventional flash memories [1]. Various types of resistive memories exist and they present improved capabilities in scalability, endurance, power consumption, operation speed and many other aspects. The mechanism responsible for their functioning has been widely studied and numerous models have been proposed but very few empirical measurements have been obtained so far. In-situ TEM is the most promising technique to obtain quantitative data at the atomic scale on a functioning device that can help us understand the dynamic mechanism responsible for their operation. However, at this time it is challenging to perform these experiments. In this presentation we will show our methodology to prepare specimens that are as close as possible to real devices in order to provide accurate measurements of the changes that are observed during resistive switching.

For in situ TEM analysis, we used a Protochips Fusion biasing holder based on patterned silicon chip. Preparation was performed by coupling a liquid Gallium Focused Ion Beam (FIB) tool and Xenon plasma FIB tool [2]. A specific method has been developed to prepare a high quality TEM foil containing a functional device. The electrical properties of ion beam deposited materials have been measured and optimized for this method. Circuit editing techniques were used to preserve the device electrical properties during TEM foil preparation and ensure good connection with the system. After a conventional lift-out, extensive micromanipulation was used to position a thick specimen onto a biasing chip. The key aspect of this preparation is to place the chunk at a controlled angle relative to the membrane (around $20^{\circ}$ ) thus allowing thinning and low energy cleaning as a final step.

Ex-situ electrical measurements were performed on a 500nm thick chunk connected to the biasing chip using the previously described preparation method (Fig.2a). We can clearly see the set and reset operation of the CBRAM sample comparable to conventional electrical characterization results obtained on the same samples [3]. We concluded that the device connected to the TEM in situ biasing chip was properly functioning, and that our FIB preparation was preserving electrical properties of the device.

The sample was then thinned to TEM foil dimensions and STEM observations were performed in a FEI Titan Themis $200 \mathrm{keV}$ (Fig.1b). STEM Energy Dispersive X-ray Spectroscopy (EDS) maps were obtained to observe the impact of resistive switching on element distribution. Deconvolution has been performed using conventional Cliff-Lorimer method and EDS maps were compared to the ones obtained on unbiased CBRAM samples. Strong modifications were detected on the $\mathrm{CuTe}_{2} \mathrm{Ge}$ layer (Fig.2b). Surprisingly, despite the extensive damage observed on the active layer after initial activation, in situ switching of the resistive memory was still possible within the TEM. For these experiments, the device was extracted and connected to the biasing chip thus removing the current selector [4]. The voltage applied to the CBRAM device for the forming step was around 3.7 V. We can conclude that the changes in element distribution and damage observed in the TEM result directly from the current surge and the associated joule effect within the chunk upon forming [5]. We observe here the failure mechanism where the $\mathrm{Cu}$ within the 
resistive layer aggregates in the device. We will also show the results of ongoing experiments on this material system.

We demonstrated that in situ TEM is a powerful tool that can be used to obtain information about structure and composition of state of the art technologies with nanometer scale resolution. In this paper we observed for the first time the impact of a CBRAM activation in situ. Solutions have been recently implemented to improve devices lifetime and optimize electrical operation within the TEM. Due to a combination of innovative specimen preparation methods and new generation in situ specimen holders, in situ TEM is now an important tool that can now be used to solve material science problems.

\section{References:}

[1] R. Waser et al, Adv. Mater. 21 (2009), p. 2632.

[2] L. Giannuzzi and N. Smith, Microsc. Microanal. 17 (2011), p. 646.

[3] J. Guy et al, IEEE Trans. Electron Devices 62 (2015), p. 3482.

[4] K. Kinoshita et al, Appl. Phys. Lett. 93 (2008), p. 033506.

[5] H. Shima et al, Appl. Phys. Lett. 93 (2008), p. 113504.
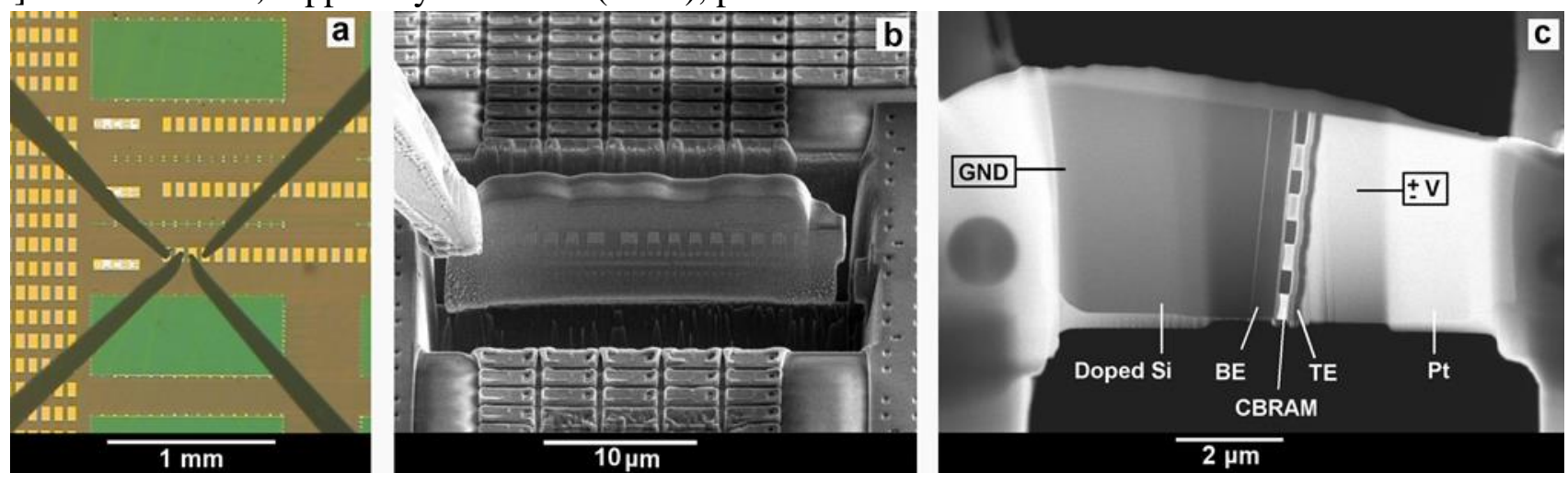

Figure 1. (a) Optical image of conventional electrical caracterisation of fully patterned wafer (b) FIB liftout of a singular device (c) individual device extracted and connected to a biasing chip for in situ TEM experiment.
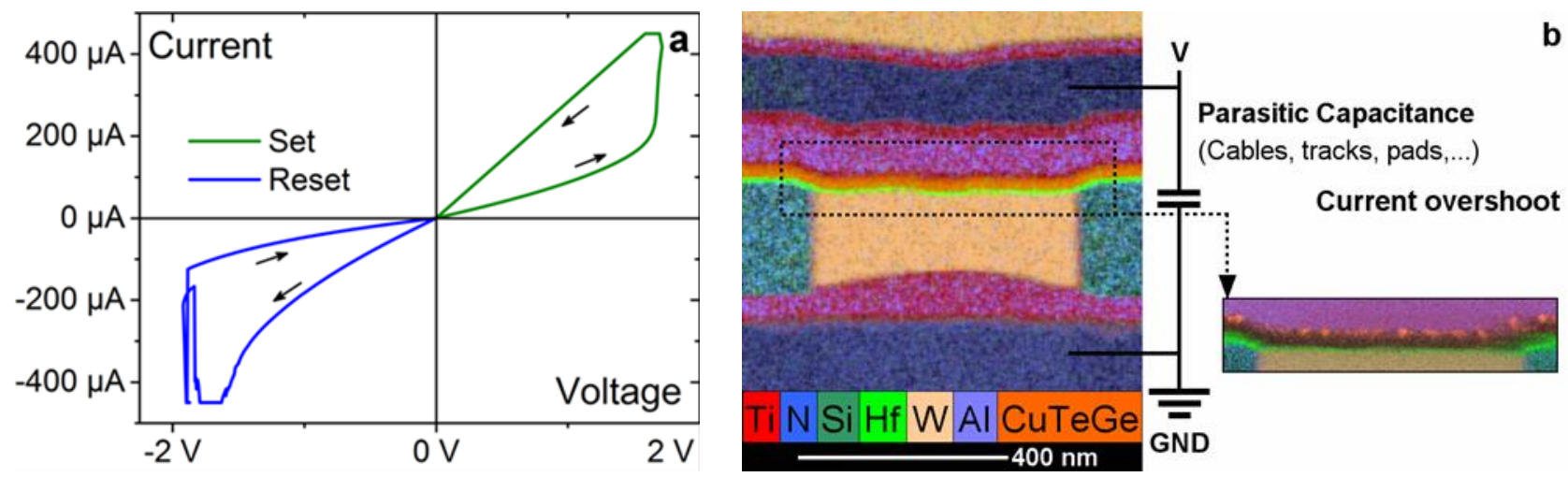

Figure 2. (a) I(V) profile of the 500nm thick connected devices obtained on the Fusion in situ TEM holder (b) EDS map of the unbiased sample (left) and biased sample (bottom right) showing extensive damage to the memory layer caused by current surge during device operation. 